\title{
Haemarthrosis due to fracture through amyloid deposits in bone in Portuguese familial amyloidosis
}

\author{
S A Allard, R H M King, P K Thomas, B E Bourke
}

Department of

Rheumatology,

St George's Hospital,

London

$S$ A Allard

B E Bourke

Department of

Neurological Science,

Royal Free Hospital

School of Medicine,

London

R H M King

P K Thomas

Dr S A Allard

Department of

Rheumatology,

West Middlesex

University Hospital,

Twickenham Road,

Isleworth,

Middlesex TW7 6AF.

Accepted for publication 6 October 1990
Correspondence to:

\begin{abstract}
A patient with Portuguese familial amyloid polyneuropathy who developed haemarthroses secondary to pathological fractures is described. Amyloid material was demonstrated on bone biopsy and confirmed immunohistochemically to be transthyretin (prealbumin). Although amyloid deposits in bone have been described in other types of amyloid, this is believed to be the first proved case of amyloid deposition resulting in pathological fracture in familial amyloidosis.
\end{abstract}

Amyloid deposits in bone have been described in association with primary amyloid, ${ }^{1}$ amyloid associated with myeloma, ${ }^{2}$ and, more recently, in amyloid associated with chronic haemodialysis. ${ }^{3}$ These deposits are seen as osteolytic lesions on $x$ ray examination and may result in pathological fractures. ${ }^{4}$ Amyloid deposits in bone have not been described in secondary amyloidosis or in the hereditary amyloidoses.

Portuguese familial amyloid polyneuropathy is an autosomal dominant condition endemic in northern Portugal. The amyloid fibril protein that accumulates in tissues is known to be a mutant form of transthyretin (formerly known as prealbumin). ${ }^{5}$ Like other types of familial amyloidosis there is a predilection for peripheral

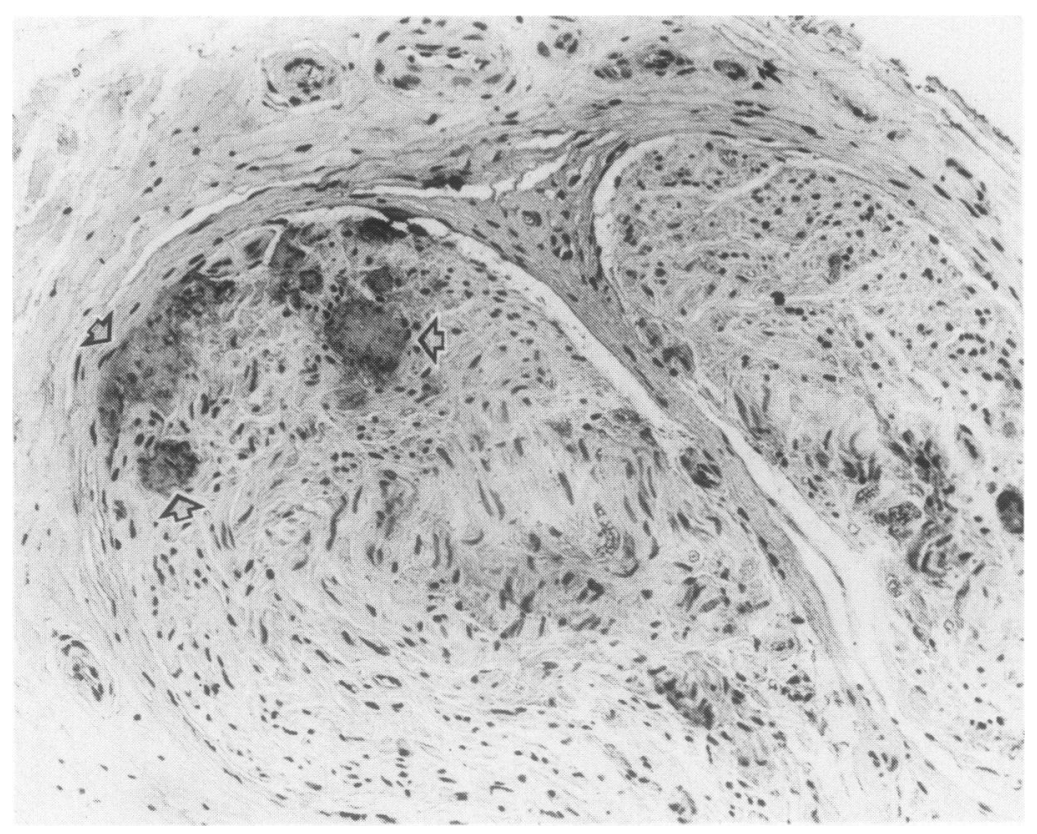

Figure 1 Sural nerve biopsy specimen showing amyloid deposits that stain positively for transthyretin (arrows), using a monoclonal antibody kindly provided by Dr Peter Collins, Ludwig Institute for Cancer Research, Stockholm. (Indirect immunoalkaline phosphatase, haematoxylin counterstained.) nerves. ${ }^{6}$ Juxta-articular collapse of bone is a rare manifestation, ${ }^{7}$ and in the few patients previously described has been attributed to bone necrosis secondary to the neuropathy. ${ }^{8-9}$

We describe a patient with Portuguese familial amyloid polyneuropathy, proved by both immunohistochemistry and cDNA analysis, who developed acutely swollen joints due to haemarthroses secondary to pathological fractures through amyloid deposits in the adjacent bone.

\section{Case report}

A 35 year old Portuguese man presented complaining of impotence, numbness in the feet and groin, diarrhoea, and weight loss. He had been referred to the rheumatology clinic because two years previously he had presented to another hospital with back pain and impotence. He had undergone a laminectomy for an L5/S1 disc protrusion, which improved the back pain but not the impotence. After this he developed diarrhoea with occasional steatorrhoea, weight loss of more than $20 \mathrm{~kg}$, increasing numbness around the external genitalia and in the feet, straining at micturition with two episodes of incontinence, and a chronic painless ulcer on the plantar surface of the right foot.

He was born in northern Portugal, where his parents and sister still live. It was noteworthy that his parents were second cousins and his sister had had weight loss and chronic diarrhoea for the previous year.

Examination showed unequal but reactive pupils, absent ankle reflexes and diminished appreciation of light touch, pinprick, and temperature sensation in both feet and over the scrotum. Sacral sensation, however, was intact, and an anal reflex was present. Dorsiflexion, eversion, and inversion at the ankle was reduced (MRC grade 4) as was flexion of the toes. There was no postural fall in blood pressure and no change in heart rate or blood pressure on Valsalva manoeuvre.

Investigations showed normal lumbar spine $x$ rays, and normal full blood count, erythrocyte sedimentation rate, and serum biochemistry. Other investigations for a peripheral neuropathy, including thyroid function, blood glucose, urinary porphyrins, Veneral Disease Research Laboratory tests, and autoimmune tests, were all normal. Nerve conduction studies showed a severe sensorimotor neuropathy. A cystogram showed an atonic bladder with a postmicturition residue and a diverticulum. Investigation of the gastrointestinal tract showed a mild abnormality of fat absorption. A rectal biopsy specimen was 


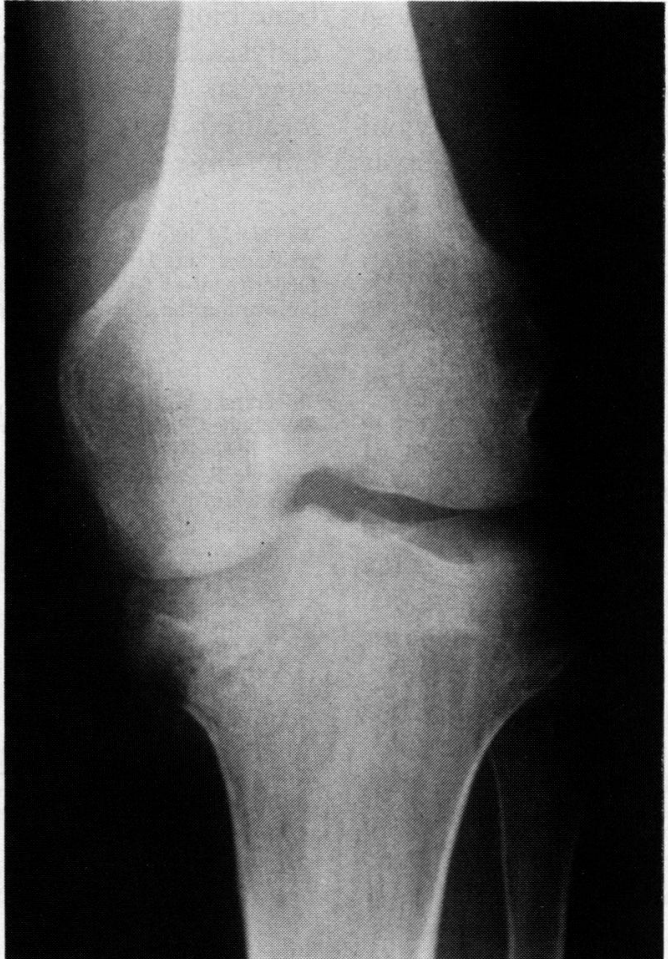

Figure 2 Radiograph of the left knee showing the pathological fracture through a lytic area beneath the medial tibial plateau.

positive for amyloid as shown by Congo red staining and polarised light microscopy. Large amyloid deposits were also demonstrated on sural nerve biopsy and were shown to be derived from transthyretin (prealbumin) on immunostaining (fig 1). Southern blot analysis confirmed the presence of abnormal bands of $5.1 \mathrm{~kb}$ and $1.5 \mathrm{~kb}$ after digestion with the restriction endonuclease NsiI as typically found

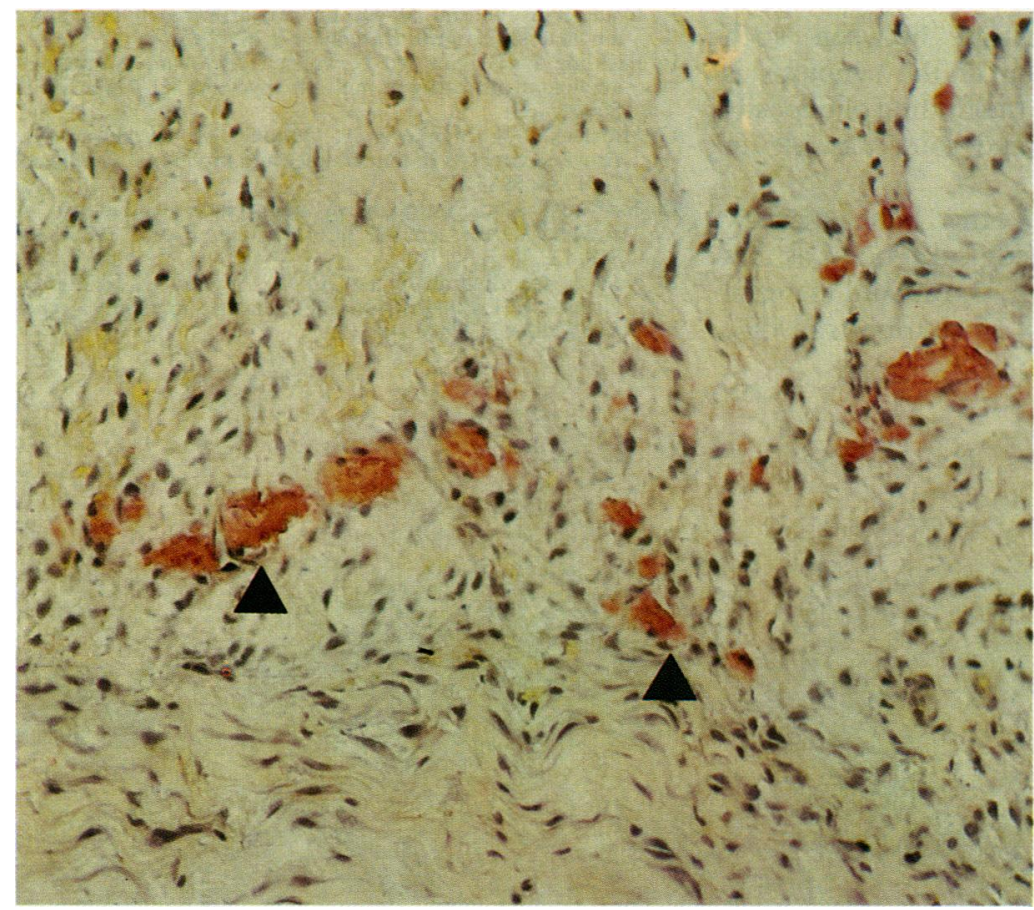

Figure 3 Within callus tissue obtained from biopsy of bone fragments of the knee, positive immunostaining for transthyretin is demonstrated here mainly within blood vessels (arrows), using the same monoclonal antibody as in fig 1. (Indirect immunoalkaline phosphatase. haematoxylin counterstained.) in Portuguese familial amyloid polyneuropathy. ${ }^{10}$

Nine months after presentation to our unit he developed an acute swelling of the left knee after running for a bus. The joint was erythematous, hot, and painful. Aspiration of the joint showed frank blood, which was sterile. Radiographs demonstrated a pathological fracture associated with a lytic area under the medial tibial plateau (fig 2). A biopsy of bone fragments from the lesion confirmed the presence of amyloid within bone and within the fracture callus. Immunohistochemical tests showed this to be transthyretin (fig 3). This fracture has healed slowly with conservative management, but he subsequently developed a swollen, erythematous but painless right ankle and forefoot. Radiographs showed collapse of the right calcaneum below the subtalar joint. He was again treated conservatively by restriction of weight bearing and the joint slowly improved.

\section{Discussion}

The description of a form of polyneuropathy in Portuguese patients characterised by the systemic deposition of amyloid with special involvement of the peripheral nerves was first made by Andrade in 1952." ${ }^{11}$ Since then many patients from multicase families have been described. ${ }^{7}$ Although classification of the hereditary amyloidoses is still based on the pattern of clinical presentation, ${ }^{6}$ recent advances in identification of the amyloid fibril proteins are likely to provide a basis for biochemical classification. ${ }^{5}$ Studies have shown that the amyloid fibril protein in Portuguese familial amyloid polyneuropathy consists of a variant of transthyretin caused by a single amino acid substitution comprising a valine for methionine at position $30 .^{12}$ The same defect has since been shown in Japanese, Brazilian, and Swedish patients with type 1 hereditary amyloidosis. ${ }^{5}$ The diagnosis can now be made by Southern blot analysis of peripheral blood using a cloned human prealbumin cDNA and the restriction endonuclease Nsil, which locates the altered codon associated with the mutant prealbumin gene. ${ }^{10}$

The presenting clinical features of the patient described in this paper are typical of Portuguese familial amyloid polyneuropathy. ${ }^{56}$ Symptoms usually appear in subjects aged 20 to 45 . In men impotence is often the first symptom, followed by a sensorimotor neuropathy affecting initially the legs, and widespread symptoms related to autonomic disturbance. Although the disorder is autosomal dominant with a high degree of penetrance his parents seem not to have been affected, though they were not formally investigated. Transthyretin gene analysis could be performed to investigate his relatives. Sporadic cases are well recognised. ${ }^{13}$ As the age of onset may not be until later life, however, children of carriers of the abnormal gene may be affected at an earlier age than their parents.

Deposits of amyloid in bone and joints have not been described in type 1 familial amyloidosis, and even carpal tunnel syndrome is said not to occur. ${ }^{6}$ Postmortem studies did not show amyloid in bone or other joint tissues despite 
widespread deposition at other sites. ${ }^{14}$ These authors suggested that the extent of bone necrosis found in Portuguese amyloid patients was directly related to the amount of amyloid deposition in nerves, and that the bone necrosis was therefore likely to be due to a Charcot-type joint, as has been described in some Japanese patients. ${ }^{8}$ A later study of this Japanese kindred describes knee and ankle joint degeneration, as in our patient. ${ }^{9}$ Again this degeneration was assumed to be neuropathic. In a large study of 483 Portuguese patients, however, bone necrosis was reported as a late clinical feature in $7 \%$ of patients. ${ }^{7}$ Although histological findings were not reported in this study, amyloid deposits in bone have apparently been found in some pathological specimens (Coutinho $\mathbf{P}$, personal communication).

Amyloid deposits in bone related to $\mathrm{AL}$ (primary or myeloma associated) amyloidosis and $\beta_{2}$ microglobulin (dialysis associated) amyloidosis are usually juxta-articular and may present with joint swelling after pathological fracture ${ }^{4}$ Localised deposits in bone resulting in osteolytic lesions are found most commonly in the proximal femur, proximal humerus, distal radius, and in the carpal bones. ${ }^{134}$ Fracture has been most frequently described in the hips, ${ }^{34}$ occurring in $23 \%$ of patients with bone cysts in dialysis associated amyloidosis. ${ }^{3}$ Fractures of the tibia and calcaneum, as seen in our patient, are extremely uncommon with any type of amyloid deposition in bone previously described.

An erosive arthritis has been reported in two patients with Swedish type 1 hereditary amyloidosis, but no amyloid material was identified in joint tissues examined. ${ }^{15} \mathrm{~A}$ synovitis that can resemble rheumatoid disease may occur in primary amyloidosis and in amyloid associated with myeloma. ${ }^{16}$ Amyloid deposits can be demonstrated in synovium and joint capsule in such patients. The relation between such deposits and the induction of the synovitis is complicated, however, by the finding of deposits of amyloid in cartilage and joint capsule in normal joints. ${ }^{17} 18$

Amyloid deposition in the bone marrow cavity seems to be a pathological process as it is not seen in the normal joint. ${ }^{18}$ Amyloid material was found in only one of 23 routine transiliac bone biopsy specimens in patients with proved dialysis associated amyloid arthropathy. ${ }^{19}$ This suggests that deposition of amyloid in bone is localised to pathological juxta-articular areas only.

We would like to thank Jane Workman for skilled technical assistance and Dr Anita Harding and Dr Ian Holt, from the Department of Clinical Neurology, Institute of Neurology, for performing the cDNA analysis.

1 Kramer M R, van Dijk J M, Hadas I, Hershko C. Destructive bone lesions in primary amyloid. Postgrad Med F 1986; 62: $1037-41$.

2 Kyle R A, Griep P R. Amyloidosis: clinical and laboratory features in 229 cases. Mayo Clin Proc 1983; 58: 665-83.

3 Bardin T, Zingraff J, Shirahama T, et al. Haemodialysisassociated amyloidosis and beta-2 microglobulin. Clinical and immunohistochemical study. Am $\mathcal{J}$ Med 1987; 83: and imm.

4 Weinfeld A, Stern M H, Marx L H. Amyloid lesions of bone. $A \nsucc R$ 1970; 108: 799-805.

5 Varga J, Wohlgethan J R. The clinical and biochemical spectrum of hereditary amyloidosis. Semin Arthritis Rheum 1988; 18: 14-28.

6 Glenner G G, Ignaczak T F, Page D L. The inherited systemic amyloidoses and localised amyloid deposits. In: Stanbury basis of inherited disease. 4th ed. McGraw-Hill: New York,
B 1978: 1308-39.

7 Coutinho P, da Silva A M, Lima J L, Barbosa A R. Forty years of experience with type 1 amyloid neuropathy. Review of 483 cases. In: Glenner G G, Costa P P, Freitas A F, eds. Amyloid and amyloidosis. Amsterdam: Exerpta Medica, Amyloid and

8 Kito S, Itoga E, Ito $\mathrm{Y}$, et al. Clinical and biochemical studies on the Ogawa village type hereditary amyloidosis with DMSO therapy. In: Glenner G G, Costa P P, Freitas A F, eds. Amyloid and amyloidosis. Amsterdam: Exerpta Medica, 1980: $153-65$.

9 Ikeda S-I, Hanyu N, Hongo M, et al. Hereditary generalised amyloidosis with polyneuropathy. Brain 1987; 110: 315-37.

10 Mita S, Maeda S, Ide M, Tsuzuki T, Shimada K, Araki S. Familial amyloidotic polyneuropathy diagnosed by cloned human prealbumin cDNA. Neurology 1986; 36: 298-301.

11 Andrade C. A peculiar form of peripheral neuropathy. Familial atypical generalised amyloidosis with special Familial atypical generalised amyloidosis with specia
involvement of peripheral nerves. Brain 1952; 75: 408-28.

12 Saraiva M J M, Birken S, Costa P P, Goodman D S. Amyloid fibril protein in familial amyloidotic polyneuropathy, Portuguese type. Definition of molecular abnormality in transthyretin (prealbumin). F Clin Invest 1984; 74: 104-19.

13 Holt I J, Harding A E, Middleton L, et al. Molecular genetics of amyloid neuropathy in Europe. Lancet 1989; i: 524-6.

14 Silva-Horta J D A, Filippe I, Durate S. Portuguese polyneuritic familial type amyloidosis. Pathol Microbiol 1964;
27: 809-25.

15 Eyanson S, Benson M D. Erosive arthritis in hereditary amyloidosis. Arthritis Rheum 1983; 26: 1145-9.

16 Hickling $P$, Wilkins $M$, Newman $G R$, et al. A study of amyloid arthropathy in multiple myeloma. $Q \mathcal{F ~ M e d ~ 1 9 8 1 ; ~}$ 50: 417-33

17 Mitrovic D R, Stankovic A, Quintero M, Ryckewaert A. Amyloid deposits in human knee and hip joints. Rheumatol Int 1985; 5: 83-9.

18 Carey N. Clinicopathological importance of deposits of amyloid in the femoral head. $f$ Clin Pathol 1985; 38 : 868-72.

19 Noel L H, Zingraff J, Bardin T, Atienza C, Kuntz D, Drueke T. Tissue distribution of dialysis amyloidosis. Clin Nephrol 1987; 27: 175-8. 